\title{
Indicaciones y resultados alejados del tratamiento quirúrgico electivo de la enfermedad diverticular del colon sigmoides
}

\author{
G uillermo Bannura C, Jaime Contreras $P$, Carlos Melo L, \\ Alejandro Barrera E, Daniel Soto C, Juan Mansilla E. \\ Indications and long term results \\ of elective surgery for sigmoid \\ diverticular disease
}

Background: There are no clear guidelines for the indication of elective surgery in sigmoid diverticular disease. Aim: To analyze the indications and long term results of elective surgery in sigmoid diverticular disease. Ma terial and Methods: Retrospective review of 100 patients (age range 25-86 years, 51 male) with sigmoid diverticular disease, operated in a lapse of 22 years. Sixty seven patients answered a survey about their disease at the end of follow up. Results: Among patients aged more than 70 years, there was a higher proportion of women. The main indication for surgery was recurrent diverticulitis in 54 patients, followed by diverticular fistula in 19. A sigmoidectomy was performed in 91 patients. Stapled anastomosis was performed in half of these patients. No patient died or required reoperation in the immediate postoperative period. During a follow up ranging from 8 to 280 months, 28 patients died for causes not associated with diverticular disease and five were lost. Those patients that answered the survey were free of symptoms related to diverticular disease and did not require new operations. Conclusions: In patients with sigmoid diverticular disease and recurrent diverticulitis or with fistulae, the long term results of surgery are satisfactory (Rev Méd Chile 2005; 133: 1037-42).

(Key Words: Colectomy; Diverticulitis; Diverticulum, colon)

Recibido el 13 de diciembre, 2004. Aceptado el 13 de mayo, 2005.

Servicio y Departamento de Cirugía, Hospital Clínico San Borja Arriarán, Campus Centro, Facultad de Medicina, Universidad de Chile.

$\mathrm{L}^{\mathrm{a}}$ a presencia de divertículos en los países industrializados se estima en alrededor de $30 \%$ de la población mayor de 60 años, de los cuales entre 10 y $25 \%$ presentará a una diverticulitis en

Correspondencia a: Dr. Guillermo Bannura C. Las Limas 1622, Las Condes. Fono: 2079582. E mail: gbannura@vtr.net algún momento de su vida ${ }^{1-3}$. Aunque no existen estadísticas de prevalencia en Chile, la enfermedad diverticular (ED) complicada es una de las principales causas de perforación de colon ${ }^{4} \mathrm{y}$, en menor grado, puede provocar cuadros de obstrucción intestinal baja y de hemorragia digestiva baja ${ }^{5,6}$. En la actualidad, persisten algunos tópicos de controversia referente a las indicaciones de cirugía electi- 
va de la ED del colon. Por una parte, la amplia variedad de las manifestaciones clínicas de la ED hacen difícil su categorización. La diverticulitis complicada, según la American Society of Colon and Rectal Surgeons (ASCRS) ${ }^{7}$, incluye pacientes con claras urgencias como la obstrucción, la peritonitis libre y el absceso pericólico o pelviano pero, también, indicaciones electivas como fístula $\mathrm{y}$ estenosis. Existe un límite impreciso entre la diverticulitis no complicada, probablemente asociada a una microperforación, y la perforación diverticular con peritonitis localizada, toda vez que la mayoría de estos cuadros tienen una excelente respuesta al tratamiento médico ${ }^{8}$. De este modo, algunas indicaciones de cirugía electiva en la ED son en gran medida empíricas y no existe medicina basada en la evidencia para apoyarlas. Por otra parte, la colopatía funcional presenta síntomas que también acompañan al paciente portador de divertículos. La confusión puede conducir a resecciones innecesarias que dan cuenta de los malos resultados funcionales que muestran algunas series extranje$\mathrm{ras}^{9}$. Aunque este tema ha sido abordado en la literatura nacional ${ }^{10,11}$, no siempre se han segregado en forma clara las indicaciones electivas de los cuadros de urgencia ${ }^{12,13}$. El motivo de esta presentación es analizar las indicaciones, el tipo de operación y los resultados inmediatos y a largo plazo de la cirugía resectiva electiva por ED del colon sigmoides.

\section{Material y MÉTOdo}

Se revisó en forma retrospectiva la base de datos de todos los pacientes intervenidos en forma consecutiva por una enfermedad diverticular del colon desde julio de 1981 a octubre de 2003. Se excluyeron los pacientes intervenidos de urgencia y los casos de enfermedad diverticular asociado con cáncer de colon. El diagnóstico de ED se basó en los antecedentes clínicos, la enema baritada, colonoscopia o ambos y el estudio de la pieza operatoria. La categorización de las indicaciones quirúrgicas se efectuó con un criterio clínico ${ }^{10}$. La técnica quirúrgica programada fue la sigmoidectomía, definiendo como límite distal justo por debajo de la unión rectosigmoidea (altura del promontorio). Se consideró como límite proximal de la resección la unión del descendente con el sigmoides. La presencia de divertículos en el colon descendente, en ausencia de signos inflamatorios, no se consideró una contraindicación para efectuar la anastomosis. En caso de compromiso del colon descendente (signos inflamatorios, engrosamiento parietal o estenosis) se extendió la resección hacia proximal, efectuando una colectomía izquierda con descenso del ángulo esplénico. La anastomosis colorrectal se realizó con poliglactina 3/0 monoplano corrido hasta 1996 y con suturas mecánicas posteriormente. El seguimiento clínico se efectuó mediante controles en el policlínico de la especialidad y contactos telefónicos, aplicándose a cada paciente una encuesta simple sobre las condiciones generales de salud, peso, nuevas hospitalizaciones, reoperaciones, hábito defecatorio y síntomas digestivos. La fecha de fallecimiento se conoció a través del sistema de informática del Registro Civil. Para el análisis estadístico se empleó el test de Student, la prueba de Wilcoxon y la prueba de proporciones para muestras independientes según corresponda, considerando significativo un p igual o menor que 0,05 .

\section{RESULTADOs}

En el período señalado se intervinieron electivamente 100 pacientes consecutivos por una ED del colon sigmoides, 51 del sexo masculino, con un promedio de edad para toda la serie de 61,1 años (extremos 25-86). El promedio de edad de los hombres fue 58 años, cifra que en las pacientes mujeres alcanzó a los 65 años $(p=0,01)$. Setenta pacientes tenían una o más enfermedades asociadas, destacando la hipertensión arterial en 37 casos, diabetes mellitus en 10, limitación crónica del flujo aéreo o tabaquismo en 15, obesidad en 9 y alcoholismo en 8. Diez y nueve pacientes habían sido sometidas a una colostomía por una complicación aguda de la ED (15 casos por perforación y 4 casos por obstrucción). El porcentaje de pacientes con el antecedente de una colostomía en el período 1981-1993 fue 42\% (16/ 38) versus $4,8 \%(3 / 62)$ en el período 1994-2003 $(p=0,0001)$. Un paciente de 77 años con el antecedente de una sigmodectomía hacía 18 años en otro centro consultó por dolor y masa palpable con estenosis infranqueable a la colonoscopia y marcada estenosis radiológica y se catalogó como 
una recidiva. Las indicaciones quirúrgicas se detallan en la Tabla 1. De los 13 pacientes con una fístula colovesical (FCV), sólo 6 tenían el antecedente de un cuadro de diverticulitis tratado médicamente. Las tres pacientes con una fístula colo-vaginal eran histerectomizadas y tampoco registraban el antecedente de diverticulitis. Hubo 20 pacientes con masa palpable, especialmente en los pacientes intervenidos por una diverticulitis recurrente o una fístula.

La enema baritada demostró divertículos con extensión variable en los 90 pacientes en que se efectuó, alteración que afectó a todo el colon en 7 $(7,7 \%)$. Este examen reveló la existencia de una FCV en 6 de los 13 casos. Mostró un trayecto hasta la piel en el único caso de fístula colo-cutánea, fue positiva en los 3 pacientes con fístula a la vagina y en uno de los dos casos de fístula colo-entérica. La cistoscopia, efectuada en 7 de los 13 pacientes portadores de una FCV fue positiva en 5 (71\%). La colonoscopia se intentó en 47 pacientes, demostrando una estenosis infranqueable en 38. La tomografía axial computada (TAC) reveló un engrosamiento difuso de la pared del colon sigmoides en los 27 pacientes en que se efectuó, una colección pericólica en 7 casos y la presencia de gas en la vejiga en los 7 pacientes con FCV en que se efectuó. De este modo, una deformación anatómica del colon sigmoides se demostró en 64 pacientes, destacando la estenosis en 40 casos, la extravasación de medio de contraste en 5 y la fístula diverticular en 19.

La intervención quirúrgica principal fue la sigmoidectomía realizada en 91 pacientes. La colectomía izquierda se efectuó en 9 casos, por compromiso inflamatorio o estenosis del colon descendente en 7 y por el antecedente de hemorragia digestiva baja recurrente originada en el colon izquierdo en 2. La anastomosis fue manual en 49 pacientes y mecánica en 51. En cuatro pacientes la anastomosis colorrectal fue protegida con una ileostomía en asa, debido al intenso compromiso inflamatorio del recto en 3 casos (uno de ellos con FCV) y por falla técnica de la anastomosis mecánica en el otro. En otros dos pacientes fue necesario realizar una resección y anastomosis de yeyuno distal por la existencia de una fístula coloentérica. El tiempo promedio de hospitalización para toda la serie fue 9,8 días (extremos 3-30). Hubo 27 complica-
Tabla 1. Indicaciones quirúrgicas electivas

\begin{tabular}{|lr|}
\hline Indicación & $\mathrm{n}(\%)$ \\
\hline Diverticulitis recurrente & 54 \\
Fístula diverticular & 19 \\
$\quad$ Colovesical & 13 \\
$\quad$ Colovaginal & 3 \\
$\quad$ Coloentérica & 2 \\
Colocutánea & 1 \\
Antecedente de perforación & 19 \\
u obstrucción por ED* & \\
Hemorragia digestiva baja recurrente & 3 \\
Diverticulitis en <40 años* & 5 \\
Total & 100 \\
\hline
\end{tabular}

*Colostomía transversa. ${ }^{* *} 3$ pacientes menores de 40 años tenían otras indicaciones.

ciones en 20 pacientes, destacando la infección de herida operatoria en 10 casos, la infección del tracto urinario en 6, el íleo prolongado en 5, un caso de sepsis de origen urinario y una fístula anastomótica de bajo débito que se maneja en forma conservadora. La morbilidad global fue $26 \%$ en el primer período y $16 \%$ en el segundo $(p=0,22)$. No hubo reoperaciones ni mortalidad operatoria.

La longitud de la pieza fijada en formalina fue, en promedio, de $25 \mathrm{~cm}$ (extremos 15-51). El informe de anatomía patológica destacó la presencia de diverticulitis y peridiverticulitis en 86 casos, con extensos focos supurados en 8, la presencia de un trayecto fistuloso con tejido granulatorio inespecífico en 8 y una reacción inflamatoria giganto-celular a cuerpo extraño en 20. No hubo elementos inflamatorios en $14 \%$ de los casos, 3 intervenidos por hemorragia, 10 por diverticulitis recurrente y 1 por FCV.

El seguimiento global de la serie fue de 80 meses como promedio (extremos 8-280) durante el cual 14 pacientes se reintervienen por una hernia incisional. En el seguimiento a largo plazo, hubo 5 pacientes perdidos y 28 fallecidos por causas no relacionadas con la ED. El grupo fallecido tenía un promedio de observación de 100 meses (extremos 14-252), sin casos de recidiva. Al corte del estudio todos los pacientes 
encuestados $(n=67)$ se encuentran asintomáticos desde el punto de vista digestivo y no se registraron casos de recidiva.

\section{DisCUSIÓN}

En nuestra serie, llama la atención el aumento significativo de pacientes en el segundo período que duplica el número del período anterior. En ausencia de estadísticas de prevalencia de la ED en el país, es difícil saber si ello representa un incremento real de la ED o interfieren otros factores, como el manejo del cuadro agudo en los servicios de urgencia. Al respecto debemos destacar dos hechos que sin duda impactan la derivación de pacientes para una cirugía electiva por ED del colon: 1) Actualmente el cuadro diverticular agudo, en la gran mayoría de los casos, se resuelve en forma satisfactoria con tratamiento conservador, en ausencia de una perforación libre u otra emergencia quirúrgica; 2) Los pacientes intervenidos como ungencia son en su mayoría sometidos a una resección sin anastomosis (operación de Hartmann) $)^{14}$, los que no han sido considerados en esta serie. Según ello, es posible que exista un aumento real de los casos de ED que requieren una cirugía electiva en nuestra área.

Actualmente existe consenso en que la distribución global por sexo es igualitaria o con leve predominio de las mujeres. Sin embargo, esta distribución no es homogénea si se estratifica por edad, existiendo un predominio de los hombres en los menores de 50 años y un predominio femenino en los mayores de 70, como se muestra en esta serie y otras ${ }^{15-17}$. En un estudio reciente de la Clínica Mayo sobre 934 pacientes (52,5\% del género femenino), la presentación clínica de la ED en promedio ocurrió en las mujeres 5 años después que en los hombres ${ }^{15}$. El predominio de los varones sería más evidente en los pacientes menores de 40 años que requieren cirugía por $\mathrm{ED}^{18}$.

La principal indicación de cirugía electiva en nuestra experiencia fue la diverticulitis recurrente en $54 \%$ de los casos, seguida de las fístulas en $19 \%$, similar a otros centros con mayor experiencia $^{15}$. No existe una categorización universal y consensuada de las indicaciones quirúrgicas electivas por una ED del colon, las que incluso cambian en series históricas provenientes de una misma institución ${ }^{11,19}$. Ello se explica porque muchos pacientes comparten una o más indicaciones. Por ello, hemos elegido mantener una clasificación que privilegia los aspectos clínicos de la ED por encima de los hallazgos de los estudios por imágenes. La diverticulitis recurrente es una indicación formal de cirugía electiva y habitualmente tiene algún grado de deformación anatómica en el examen radiológico, lo que facilita la decisión terapéutica. La masa palpable es muy difícil que no tenga un correlato en el estudio radiológico contrastado o en la TAC.

La incidencia de fístula diverticular de 19\% observada en nuestra serie es alta comparada con otros estudios nacionales ${ }^{11,12}$ o extranjeros s $^{2,8,9,15}$ que fluctúan entre 4 y $8 \%$, lo que probablemente refleja la demora en el diagnóstico y el tratamiento de la ED. Es llamativo la ausencia de una crisis grave de diverticulitis en $45 \%$ de nuestros pacientes portadores de una FCV. Por tratarse de trayectos cortos y pequeños, la sensibilidad de la enema baritada no sobrepasa $60 \%$ (45\% en nuestra experiencia), mientras que la TAC muestra aire en la vejiga en $96 \%$ de los $\operatorname{casos}^{2,20,21}$. Esta complicación es más común en el paciente varón ${ }^{15}$, lo que podría explicarse por el efecto protector del útero en la mujer.

Aunque no hay evidencia definitiva sobre el tema, un episodio de diverticulitis que responde satisfactoriamente al tratamiento médico en el paciente menor de 40 años, sería para algunos autores una indicación formal de cirugía electiva por ED, considerando que la enfermedad en estos casos tiene un curso más agresivo ${ }^{17,22-24}$.

El prolongado seguimiento de nuestros pacientes, nos ha permitido corroborar que la sigmoidectomía es la intervención de elección para tratar la ED del colon sigmoides, considerando como límite distal la unión rectosigmoidea. El punto de referencia anatómico es inmediatamente por debajo del promontorio, con lo cual la anastomosis queda entre 13 y $15 \mathrm{~cm}$ del margen anal. La conservación de parte del sigmoides distal favorece la recidiva de la enfermedad e incrementa el riesgo de una re-resección ${ }^{25,26}$. La colectomía izquierda fue necesaria en sólo $9 \%$ de nuestros pacientes, a diferencia de otras experiencias nacionales $^{11}$. Compartimos con otros autores que la presencia de divertículos en el segmento a anasto- 
mosar en ausencia de engrosamiento parietal 0 signos inflamatorios no implica la necesidad de extender la resección hacia proximal2,7,15,27.

El estudio histopatológico de la pieza resecada confirmó la ausencia de compromiso inflamatorio en $14 \%$ de los casos, cifra que en la literatura fluctúa entre 15 y 30\%8,19,28. El análisis más cercano de estos pacientes muestra que la indicación de cirugía programada fue en 3 casos la hemorragia digestiva baja a repetición, en los cuales la ausencia de signos inflamatorios es un hecho habitual ${ }^{28}$. El resto había sufrido repetidos episodios de diverticulitis, dos habían sido intervenidos de urgencia por una peritonitis localizada y uno tenía una fístula colovesical, cuya única explicación posible es la capacidad de reparación del fenómeno inflamatorio en una enfermedad que es esencialmente extracolónica. Sin embargo, los resultados funcionales alejados en estos pacientes no difieren del resto y no hemos observado nuevos episodios de diverticulitis ni han requerido nuevas intervenciones. La adecuada selección de los pacientes y probablemente lo avanzado de la ED en la mayoría de nuestros pacientes, explicaría la ausencia de síntomas residuales que en algunas series se eleva a $30 \% 8,29,30$.

\section{REFERENCIAS}

1. PARKS TG. Natural history of diverticular disease of the colon. Clin Gastroenterol 1975; 4: 53-69.

2. Young-Fadok TM, Roberts PL, Spencer MP, WolfF BG. Colonic diverticular disease. Curr Probl Surg 2000; 7: 457-516.

3. SARIN S, Boulos PB. Long-term outcome of patients presenting with acute complications of diverticular disease. Ann R Coll Surg Engl 1994; 76: 117-20.

4. Bannura G, Vera E, Vargas M. Cirugía de urgencia en la enfermedad diverticular del colon complicada con perforación. Rev Chil Cir 1991; 43: 1558 .

5. Bannura G. Etiología y pronóstico de la obstrucción mecánica aguda del colon: Revisión de 352 casos. Rev Chil Cir 1993; 45: 397-404.

6. Bannura G, Barrera A, Melo C, Contreras J, Soto D. Hemorragia digestiva baja masiva: resultados
En conclusión, en la mayoría de los casos las indicaciones quirúrgicas electivas de la ED son claras y conducen a buenos resultados funcionales en el seguimiento a largo plazo. La cirugía programada en menores de 40 años, luego de un episodio de diverticulitis, pareciera estar indicada por la mayor gravedad del cuadro en estos pacientes, habitualmente obesos. Extender este criterio a los menores de 50 años es controversial y la decisión deberá considerar cada paciente por separado en el contexto de las eventuales enfermedades asociadas o condiciones como la inmunosupresión. La intervención de elección es la sigmoidectomía, cuyo límite distal debiera ser por debajo de la unión rectosigmoidea. No es necesario extender la resección proximal para eliminar todo el colon afectado por divertículos en ausencia de engrosamiento parietal o signos inflamatorios. En nuestra experiencia la sigmoidectomía se puede realizar a través de una pequeña laparotomía con baja morbilidad y sin mortalidad con la técnica tradicional, lo que es comparable con estudios prospectivos multicéntricos efectuados en Europa $^{31}$. La resección por vía laparoscópica es una alternativa atractiva que permitiría reducir el dolor postoperatorio, disminuir los días de hospitalización y lograr un reintegro laboral precoz ${ }^{32}$.

del estudio y tratamiento en 20 pacientes consecutivos. Rev Méd Chile 2002; 130: 869-78.

7. Roberts P, Rosen L, Cirocco W, Fleshman J, LefF E, LeviEN D ET AL. Practice parameters for sigmoid diverticulitis, supporting documentation. The Standards Task Force, American Society of Colon and Rectal Surgeons (ASCRS). Dis Colon Rectum 1995; 38: 126-32.

8. Thompson DA, BAiley HR. Management of acute diverticulitis with abscess. Sem Colon Rectal Surg 1990; 1: 74-80.

9. Breen RE, Corman ML, Robertson WG, Prager ED. Are we really operating on diverticulitis? Dis Colon Rectum 1986; 29: 174-6.

10. Uribe S, Bannura G, Contreras J, Portaler P, Sabat J. Cirugía resectiva electiva de la enfermedad diverticular del colon. Rev Chil Cir 1996; 48: 179-83.

11. ZÚÑIGA A, Viñueia E, López F, Rahmer A. Enfermedad diverticular del colon. Resultados del tratamiento quirúrgico electivo. Rev Chil Cir 2001; 53: 342-6. 
12. Jensen C, Pérez G, Garrido R, Bocic G, Azolas C, ABEDRAPO $M$ ET aL. Enfermedad diverticular del colon. Análisis de 144 casos operados. Rev Chil Cir 2000; 52: 123-8.

13. SuÁreZ J. Cirugía de la enfermedad diverticular del colon complicada. Rev Chil Cir 1990; 42: 253-6.

14. BANNURA G. Rol actual de la operación de Hartmann: análisis crítico. Rev Chil Cir 2002; 54: 555-60.

15. McConnell EJ, Tessier DJ, Wolff BG. Populationbased incidence of complicated diverticular disease of sigmoid colon based on gender and age. Dis Colon Rectum 2003; 46: 1110-4.

16. Tudor RG, Farmakis N, Keighley MRB. National audit of complicated diverticular disease: analysis of index cases. Br J Surg 1994; 81: 730-2.

17. Makela J, Vuolo S, Kiviniemi H, Laitnen S. Natural history of diverticular disease: when to operate? Dis Colon Rectum 1998; 41: 1523-8.

18. Acosta JA, Grebenc ML, Doberneck RC, McCarthy JD, FRY DE. Colonic diverticular disease in patients 40 years old or younger. Am Surg 1992; 58: 605-7.

19. Botinew A, López F, RAhMER A, ZÚNIIGA A. Enfermedad diverticular del colon, indicaciones y resultados del tratamiento quirúrgico electivo. Rev Méd Chile 1990; 118: 1206-10.

20. JARRETT TW, Vaughan TD JR. Accuracy of computarized tomography in the diagnosis of colovesical fistula secondary to diverticular disease. J Urol 1995; 153: 44-6.

21. Bannura G, Cabelo R, Riffo C. Fístulas colovesicales en adultos: análisis de 22 casos. Rev Chil Urol 1997; 62: 247-51.

22. Cunningham MA, Davis JW, KaUPs KL, Medical versus surgical management of diverticulitis in patients under age 40. Am J Surg 1997; 174: 733-6.
23. Vignati PV, Welch JP, Cohen JL. Long-term management of diverticulitis in young patients. Dis Colon Rectum 1995; 38: 627-9.

24. Spivak H, Weinrauch S, Harvey JC, Surick B, FERSTENBERg H, Friedman I. Acute colonic diverticulitis in young patients. Dis Colon Rectum 1997; 40: 570-4.

25. Benn PL, Wolfr BG, ILSTRup DM. Level of anastomosis and recurrent colonic diverticulitis. Am J Surg 1986; 151: 269-71.

26. BeLL AM, Wolff BG. Progression and recurrence after resection for diverticulitis. Sem Colon Rectal Surg 1990; 1: 99-102.

27. Wolf BG, Ready RL, MacCarthy RL, Dozois RR, BEART RW JR. Influence of sigmoid resection on progression of diverticular disease of the colon. Dis Colon Rectum 1984; 27: 645-7.

28. Morson BC. The muscle abnormality in diverticular disease of the sigmoid colon. Br J Radiology 1970; 2: 136-8.

29. Charnock FML, Rennie JR, Welwood JM, Todd IP. Results of colectomy for diverticular disease of the colon. Br J Surg 1977; 64: 417-9.

30. Munson KD, Hensien MA, Jacob LN, Robinson AM, LISTON WA. Diverticulitis: a comprehensive followup. Dis Colon Rectum 1996; 39: 318-22.

31. Pessaux P, Muscari F, Oueluet JF, Msika S, Hay JM, Mшшат B ET AL Risk factors for mortality and morbidity after elective sigmoid resection for diverticulitis: prospective multicenter multivariate analysis of 582 patients. World J Surg 2004; 28: 92-6.

32. Stevenson ARL, Stitz RW, Lumley JW, Fielding GA. Laparoscopically assited anterior resection for diverticular disease. Follow-up of 100 consecutive patients. Ann Surg 1998; 227: 335-42. 